\title{
Intravitreal bevacizumab has initial clinical benefit lasting eight weeks in eyes with neovascular age-related macular degeneration
}

\author{
P William Conrad \\ David N Zacks \\ Mark W Johnson \\ Department of Ophthalmology \\ and Visual Sciences, Kellogg Eye \\ Center, University of Michigan, \\ Ann Arbor, Michigan, USA
}

Correspondence: Mark W Johnson Retina Service, Kellogg Eye Center, University of Michigan, 1000 Wall Street, Ann Arbor, MI 48I05, USA

Tel +I 7349367760

Emailmarkwj@med.umich.edu
Purpose: To determine whether the effect of a single initial intravitreal injection of bevacizumab for neovascular age-related macular degeneration (AMD) persists for 8 weeks.

Methods: We reviewed the records of 25 consecutive patients with neovascular AMD treated with intravitreal bevacizumab. Patients were included $(n=15)$ if follow up data were available from 4 and 8 week visits after a single initial injection. Additionally, optical coherence tomography (OCT) images were graded qualitatively in a masked fashion by a single reader.

Results: Baseline mean visual acuity was $20 / 200$, improving to $20 / 125$ at 4 weeks ( $p=0.0153$ ) and $20 / 100$ at 8 weeks $(p=0.0027)$. Mean central retinal thickness was $316 \pm 107 \mu \mathrm{m}$ at baseline and decreased to $223 \pm 70 \mu \mathrm{m}$ and $206 \pm 45 \mu \mathrm{m}$ at 4 and 8 weeks post-injection, respectively $(\mathrm{p}=0.0003$ and 0.0005$)$. By masked OCT grading, macular fluid was resolved in 10/15 (66.7\%) and 11/15 (73.3\%) eyes at 4 and 8 weeks, respectively, and 3/15 (20\%) eyes had continued reduction in residual macular fluid between 4 and 8 weeks.

Conclusions: A single initial bevacizumab injection has persistent clinical benefit lasting 8 weeks in most eyes with neovascular AMD. Results of prospective randomized studies are needed before changes in treatment regimens can be recommended.

Keywords: age-related macular degeneration, bevacizumab, choroidal neovascular membrane, optical coherence tomography

\section{Introduction}

Vascular endothelial growth factor (VEGF) is a mediator of many pathologic conditions, including neovascular age-related macular degeneration (AMD) (Lopez et al 1996; Kvanta et al 1996). Pegaptanib sodium (OSI/Eyetech, Melville, NY), an aptamer that targets the 165 amino-acid isoform of VEGF, was the first anti-VEGF agent approved for the treatment of neovascular AMD. Intravitreal pegaptanib administered every six weeks was shown to slow the rate of vision loss compared with a shaminjected control group after one year (Gragoudas et al 2004). More recently, intravitreal injections of the anti-VEGF reagents, ranibizumab (Lucentis; Genentech, Inc., South San Francisco, CA) and bevacizumab (Avastin; Genentech, Inc.) have been shown to result in improvement in mean visual acuity in treated eyes (Avery et al 2006; Heier et al 2006). Ranibizumab and bevacizumab were genetically engineered from the same murine monoclonal antibody against VEGF-A, the angiogenic protein considered to be the primary driver of choroidal neovascular membranes (Aiello et al 1995; Rakic et al 2003; Matsuoka et al 2004). Ranibizumab is a humanized antigen-binding fragment (Fab) against VEGF and was approved by the United States Food and Drug Administration (FDA) in June, 2006 based upon phase III MARINA and ANCHOR trials which confirmed its safety and efficacy (Brown et al 2006; Rosenfeld et al 2006a). Bevacizumab is a full length, humanized monoclonal antibody against VEGF 
and is FDA-approved for the treatment of metastatic colon cancer by intravenous use. The off-label use of intravenous bevacizumab for the treatment of neovascular AMD was initially reported by Michels and colleagues (Michels et al 2005). Subsequently, uncontrolled, retrospective and prospective case series have demonstrated short-term efficacy of intravitreal bevacizumab in neovascular AMD (Rosenfeld et al 2005; Avery et al 2006; Bashshur et al 2006; Rich et al 2006; Spaide et al 2006).

While growing evidence supports the use of off-label intravitreal bevacizumab in the treatment of exudative AMD, little is known about the optimal dosing paradigm for this antibody. Typically, clinicians have followed patients monthly after the initial dose of bevacizumab and re-injected for persistent cystic maculopathy and/or sub-retinal fluid by clinical examination and optical coherence tomography (OCT) imaging (Avery et al 2006; Bashshur et al 2006; Rich et al 2006). There is little scientific evidence to support this strategy. Rather it evolved from the dosing regimen employed in the ranibizumab clinical trials (Heier et al 2006; Rosenfeld et al 2006b). In the ANCHOR and MARINA trials, patients randomized to the ranibizumab arm received monthly injections for the duration of the trial (Brown et al 2006; Rosenfeld et al 2006a). However, the fact that bevacizumab (149 kDa) and ranibizumab (48 kDa) have different molecular weights presumably alters their pharmacokinetic behavior when given intravitreally. Mordenti and colleagues (1999) showed in nonhuman primates that trastuzumab (Herceptin; Genentech, Inc), a full-length antibody similar to bevacizumab, has a vitreous half-life of 5.6 days while an antibody Fab similar to ranibizumab has a half-life of 3.2 days. Given the increased half-life of the full sized antibody, it is reasonable to suspect that bevacizumab requires less frequent dosing than ranibizumab. We hypothesized that the effects of an initial bevacizumab injection would persist beyond the 4 week period when patients have typically been reexamined and reinjected for persistent exudation. We therefore tested whether the clinical effects of a single initial intravitreal bevacizumab injection persist for 8 weeks in eyes with neovascular AMD.

\section{Methods}

We retrospectively reviewed the medical records of consecutive patients with neovascular AMD treated with intravitreal bevacizumab by one of the authors (MWJ). Between November, 2005 and June, 2006, 25 patients received an initial injection of bevacizumab as part of their clinical care for active neovascular AMD. Disease activity was detected on biomicroscopic examination and confirmed by fluorescein angiography and OCT, with indocyanine angiography as needed to characterize retinal angiomatous proliferation (RAP) lesions. Approval to perform the retrospective review was obtained from our Institutional Review Board.

The standard clinical treatment regimen employed during the time period of this retrospective study was two induction injections given two months apart, followed by subsequent reinjection as needed for recurrent exudation. Following the initial injection, patients were not routinely retreated earlier than two months, even if cystoid macular edema, subretinal fluid or subretinal pigment epithelial (RPE) fluid persisted at one month. Patients $(n=15)$ were included in this analysis if complete follow up data, including Snellen visual acuity and OCT, were available from 4 and 8 week visits after the initial injection. No patient included in this study was re-injected with bevacizumab or received any other AMD intervention prior to their 8 week post-injection visit.

Patients were informed of the off-label use of intravitreal bevacizumab (Avastin; Genentech, Inc.) and received a thorough discussion of all risks, potential benefits and alternative treatment options. Aliquots $(0.12 \mathrm{ml})$ of commercially available bevacizumab (Avastin) were placed in a tuberculin syringe by a compounding pharmacy using aseptic technique. Aliquots were stored at $4{ }^{\circ} \mathrm{C}$ until used (within 14 days of preparation). Following topical antibiotic drops and anesthesia with proparacaine and 4\% lidocaine, the eye was cleaned with $10 \%$ povidone-iodine and draped in a sterile fashion. A lid speculum was placed in the eye. Bevacizumab ( $1.25 \mathrm{mg}$ in $0.05 \mathrm{cc}$ ) was injected through a 30 gauge needle introduced into the vitreous cavity $3.5-4.0 \mathrm{~mm}$ from the limbus. Topical antibiotic was applied and the intraocular pressure was monitored.

Patients returned to the clinic at 4 and 8 weeks following injection with bevacizumab. At these visits, Snellen visual acuity was measured and fundus biomicroscopy and OCT imaging were performed. OCT imaging included 6 diagonal, fast, low-density 6-mm scans performed at 30 degree intervals. The average thickness of the central 1-mm subfield was determined from the fast macular thickness maps calculated from the 6 low-resolution diagonal scans. No attempt was made retrospectively to adjust for improper retinal boundaries determined by automated OCT analysis. In addition, two high-density, high-resolution scans in a cross-hair configuration were obtained and used to qualitatively evaluate the degree of macular exudation at the baseline, 4 and 8 week visits. As part of this retrospective study, OCT images were graded by a single reader masked to the identity of the patient, 
visit date and mean central retinal thickness measurements. Macular fluid (defined as cystoid edema and/or subretinal fluid) and sub-RPE fluid were analyzed separately for each visit. The baseline OCT was identified and compared to the masked 4 and 8 week post-injection OCTs. Each set was graded as to whether the fluid components were resolved, improved (but not resolved), unchanged or worsened.

Statistical comparisons of mean central thickness measurements and $\log$ MAR visual acuities were performed using two-tailed paired t-tests (SAS, Version 9.1, Cary, NC). A $p$ value of $<0.05$ was considered to be statistically significant.

\section{Results}

Fifteen eyes of fifteen patients met the inclusion criteria for this retrospective study; none of these patients were excluded from analysis. Mean patient age was 79.7 years (range, 63-89). There were seven men (46.7\%) and eight women (53.3\%). Seven of fifteen (46.7\%) eyes had received previous treatment for exudative AMD including laser photocoagulation (3), photodynamic therapy with verteporfin (3) and pegaptanib (2). Choroidal neovascular membranes were characterized as occult-only in 6/15 (40\%) eyes, predominantly classic in $2 / 15(13.3 \%)$ eyes and minimally classic in $2 / 15(13.3 \%)$ eyes. Five of fifteen (33.3\%) eyes had retinal angiomatous proliferation lesions.

Table 1 shows mean visual acuity and central retinal thickness measurements. Compared with baseline values of 20/200 (logMAR $0.98 \pm 0.44)$, mean visual acuity improved by 4 weeks to $20 / 125(\log$ MAR $0.76 \pm 0.35, p=0.0153)$. Without additional bevacizumab injections, the visual improvement was maintained through 8 weeks, with a mean visual acuity of 20/100 (logMAR $0.70 \pm 0.34, p=0.0027)$. At the 4 week follow-up, three eyes (20\%) had an improvement in visual acuity of at least 3 lines and at week 8 , five eyes $(33.3 \%)$ had at least a 3-line improvement. Similar results were found when comparing the mean central retinal thickness. Mean central retinal thickness was $316 \pm 107 \mu \mathrm{m}$ at baseline and decreased to $223 \pm 70 \mu \mathrm{m}(\mathrm{p}=0.0003)$ and
$206 \pm 45 \mu \mathrm{m}(\mathrm{p}=0.0005)$ at 4 and 8 weeks post-injection, respectively (Table 1 ).

Figures 1A and 1B show the distribution of individual patients with respect to visual acuity at baseline and at 4 weeks or 8 weeks, respectively. Following a single bevacizumab injection, stable (within 1 Snellen line) or improved vision was seen in all $15(100 \%)$ eyes at 4 and 8 weeks. Without additional injections, the visual acuity remained stable or improved further between 4 and 8 weeks in all eyes (Figure 1C).

Figures $2 \mathrm{~A}$ and $2 \mathrm{~B}$ plot the distribution of individual patients with respect to central retinal thickness measurements at baseline and at week 4 or week 8 , respectively. Compared with baseline, stable (within $50 \mu \mathrm{m}$ ) or decreased central thickness was seen in all $15(100 \%)$ eyes by 4 weeks (Figure 2A). These results persisted at 8 weeks (Figure 2B). Between 4 and 8 weeks, central retinal thickness measurements were stable (within $50 \mu \mathrm{m}$ ) in 11/15 (73.3\%) eyes, improved in $3(20 \%)$ eyes, and worsened in $1(6.7 \%)$ eye (Figure 2C). In the lone eye that showed worsening between 4 and 8 weeks, the visual acuity remained stable during this period.

Results of the masked evaluation of OCT images are presented in Table 2. Following a single initial intravitreal bevacizumab injection, macular fluid (cystoid edema and/or subretinal fluid) was resolved in 10/15 (66.7\%) patients at 4 weeks and 11/15 (73.3\%) patients at 8 weeks. Masked comparison of the 4 and 8 week OCT images showed that 3/15 $(20 \%)$ patients had continued reduction in residual macular fluid between these time points (Table 2, Figure 3). The macular fluid was graded as worse at 8 weeks compared with 4 weeks in only $1(6.7 \%)$ patient, and was unchanged in the remaining 11 (73.3\%) patients (Figure 4). Six of the 15 patients in our study had a serous pigment epithelial detachment (PED) component at the pre-injection visit. The response of the sub-RPE fluid in these eyes was similar to that of the cystoid macular edema and subretinal fluid. The serous RPE detachment had resolved in $4 / 6(67.7 \%)$ patients at both the 4 and 8 week post-injection visits (Table 2). Between 4 and 8 weeks, 1/6 (16.7\%) patients

Table I Mean visual acuity and central retinal thickness following a single bevacizumab injection $(\mathrm{n}=15)$

\begin{tabular}{llll}
\hline & Baseline & Week 4 & Week 8 \\
\hline Mean Va \pm SD (logMAR) & $0.98 \pm 0.44$ & $0.76 \pm 0.35(p=0.0153)$ & $0.70 \pm 0.34(P=0.0027)$ \\
Mean Va (Snellen) & $20 / 200$ & $20 / 125$ & $20 / 100$ \\
$\geq 3$ line improvement in Va & NA & $3 / 15(20 \%)$ & $5 / 15(33.3 \%)$ \\
Mean central retinal thickness \pm SD $(\mu \mathrm{m})$ & $316 \pm 107$ & $223 \pm 70(P=0.0003)$ & $206 \pm 45(P=0.0005)$ \\
\hline
\end{tabular}

Abbreviations: Va, visual acuity; logMAR, logarithm of minimum angle of resolution; NA, not applicable; SD, standard deviation. 
A.

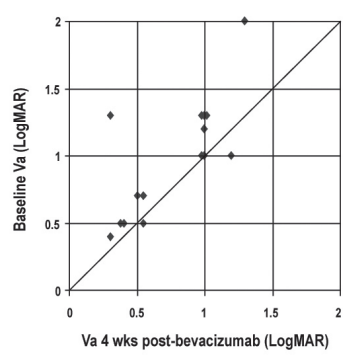

B.

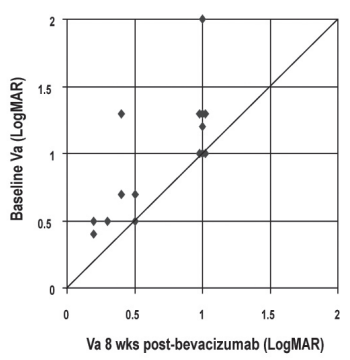

C.

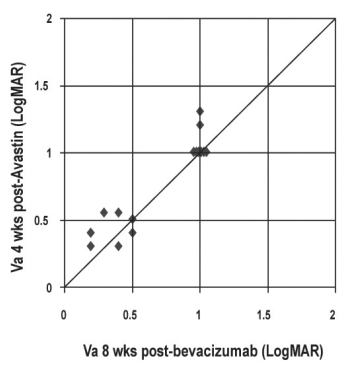

Figure I Distribution of visual acuity (logMAR) in 15 eyes between baseline, 4 and 8 weeks following a single bevacizumab injection. Interval improvement in vision is represented by points above the line. A) Baseline versus 4 weeks. B) Baseline versus 8 weeks. C) 4 weeks versus 8 weeks.

had further resolution of residual sub-RPE fluid, while the remaining patient demonstrated no change in residual sub-RPE fluid. No eyes showed an increase in sub-RPE fluid during this time interval.

\section{Discussion}

Intravitreal bevacizumab has been shown to be effective in the management of exudative AMD (Rosenfeld et al 2005; Avery et al 2006; Bashshur et al 2006; Rich et al 2006; Spaide et al 2006). While ranibizumab has become the gold standard for neovascular AMD since its approval by the FDA, off-label use of bevacizumab remains widespread, owing in part to cost considerations. Cost aside, some patients and clinicians might be inclined to use bevacizumab if certain therapeutic properties, such as duration of action, are shown in future clinical trials to be advantageous compared with ranibizumab. Because bevacizumab is approximately three times larger than ranibizumab, and because previous studies have demonstrated a 75\% increase in the intravitreal half-life of a full-length antibody compared to an antibody fragment, we hypothesized that the effects of an initial dose of intravitreal bevacizumab would persist for 8 weeks in most eyes.

The anatomical and visual response to a single injection of intravitreal bevacizumab in our patients appear to support

this hypothesis. The mean central retinal thickness decreased from $316 \mu \mathrm{m}$ at baseline to $223 \mu \mathrm{m}$ at 4 weeks post-injection. Without further bevacizumab injections, there was a trend toward continued improvement in mean central retinal thickness to $206 \mu \mathrm{m}$ between 4 and 8 weeks. Similarly, mean Snellen visual acuity improved from 20/200 at baseline to 20/125 at 4 weeks and 20/100 at 8 weeks.

Importantly, masked qualitative evaluation of highresolution OCT scans confirmed the anatomical response suggested by automated central retinal thickness measurements. We performed the qualitative analysis because of the inherent limitations of automated quantitative OCT thickness measurement in the complex macular anatomy of neovascular AMD. As emphasized by previous authors (Sadde et al 2006), retinal thickness measurement errors occur frequently in AMD with current Stratus OCT analysis algorithms, in part because of poor patient fixation, difficulty in determining anterior and posterior retinal boundaries, and the inability to take into account the presence of sub-RPE fluid. Because of these limitations, qualitative evaluation of OCT scans is a more accurate method of assessing the macular anatomic response to anti-VEGF therapy.

In our study, masked qualitative analysis of OCT scans showed that the macular fluid (macular edema and/or subretinal fluid) status was stable or showed continued improvement in 14/15 (93\%) of patients between 4 and 8 weeks following a

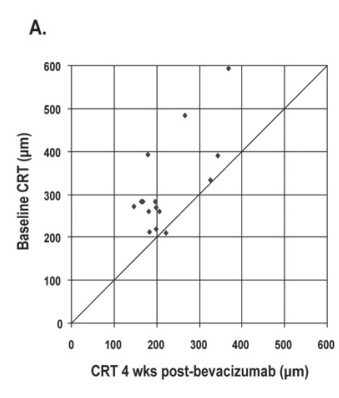

B.

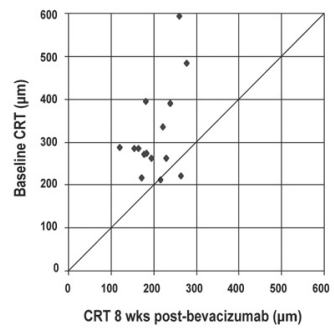

c.

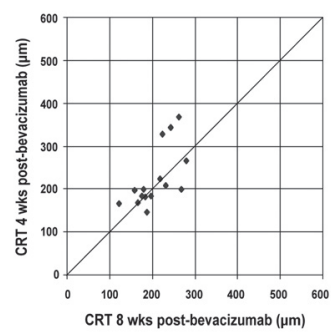

Figure 2 Distribution of central I-mm retinal thickness (CRT) at baseline, 4 and 8 weeks following a single bevacizumab injection. Decreased central retinal thickness is represented by points above the line. A) Baseline versus 4 weeks. B) Baseline versus 8 weeks. C) 4 weeks versus 8 weeks. 
Table 2 Masked grading of OCT images at baseline, 4 , and 8 weeks

\begin{tabular}{|c|c|c|c|c|c|c|}
\hline & \multicolumn{3}{|c|}{ Macular fluid ( 15 eyes) } & \multicolumn{3}{|c|}{ Sub-RPE fluid (6 eyes) } \\
\hline & $\begin{array}{l}\text { Baseline to } \\
4 \text { weeks n (\%) }\end{array}$ & $\begin{array}{l}\text { Baseline to } \\
8 \text { weeks n (\%) }\end{array}$ & $\begin{array}{l}4 \text { weeks to } \\
8 \text { weeks n (\%) }\end{array}$ & $\begin{array}{l}\text { Baseline to } \\
4 \text { weeks n (\%) }\end{array}$ & $\begin{array}{l}\text { Baseline to } \\
8 \text { weeks n (\%) }\end{array}$ & $\begin{array}{l}4 \text { weeks to } \\
8 \text { weeks n (\%) }\end{array}$ \\
\hline Resolved & $10(66.7 \%)$ & II (73.3\%) & NA & $4(66.7 \%)$ & $4(66.7 \%)$ & NA \\
\hline Improved & $3(20 \%)$ & $3(20 \%)$ & $3(20 \%)$ & I (I6.7\%) & $2(33.3 \%)$ & I (I6.7\%) \\
\hline Unchanged & $2(13.3 \%)$ & I (6.7\%) & II (73.3\%) & I (I6.7\%) & 0 & $5(83.3 \%)$ \\
\hline Worsened & 0 & 0 & I (6.7\%) & 0 & 0 & 0 \\
\hline
\end{tabular}

Abbreviations: $\mathrm{OCT}$, optical coherence tomography; RPE, retinal pigment epithelium; NA, not applicable.

single bevacizumab injection. The remaining patient showed a mild increase in macular fluid during this time period. Similarly, by masked grading, sub-RPE fluid status between 4 and 8 weeks continued to improve or was stable in $6 / 6$ eyes $(100 \%)$ with a serous RPE detachment component at baseline. Using similar qualitative OCT analysis, Costa and colleagues (2006) found in a phase I dose-escalation study that macular architecture was stable or improved from baseline in $91.1 \%$ of eyes at 6 weeks and $83.7 \%$ of eyes at 12 weeks following a single initial bevacizumab injection for exudative AMD. Within each dose group, more eyes demonstrated favorable macular remodeling on angiographic and OCT evaluations at 6 weeks compared with 12 weeks, suggesting a decline in biologic activity during this time period.

These data suggest that bevacizumab can be initially dosed in most eyes at intervals of 6 to 8 weeks. The observation that a subset of eyes shows continued improvement in macular and/or sub-RPE fluid between 4 and 8 weeks after a single injection and that fluid reaccumulation rarely occurs during this period suggests that a pharmacologic effect initially persists in the eye for approximately two months. Reinjection of bevacizumab for persistent macular or sub-RPE fluid at 4 weeks is probably unnecessary in most cases, since continued resolution of fluid over the subsequent month may occur without reinjection.
Horizontal

A.

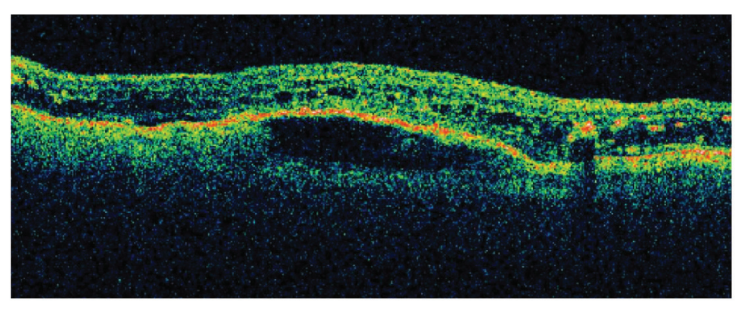

B.

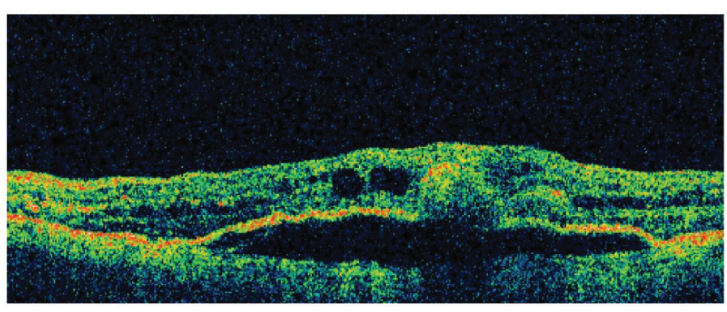

C.

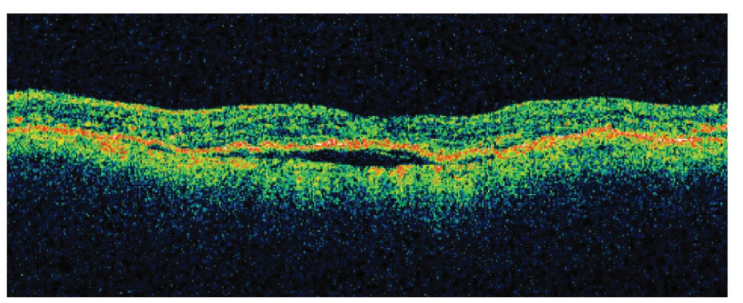

Vertical
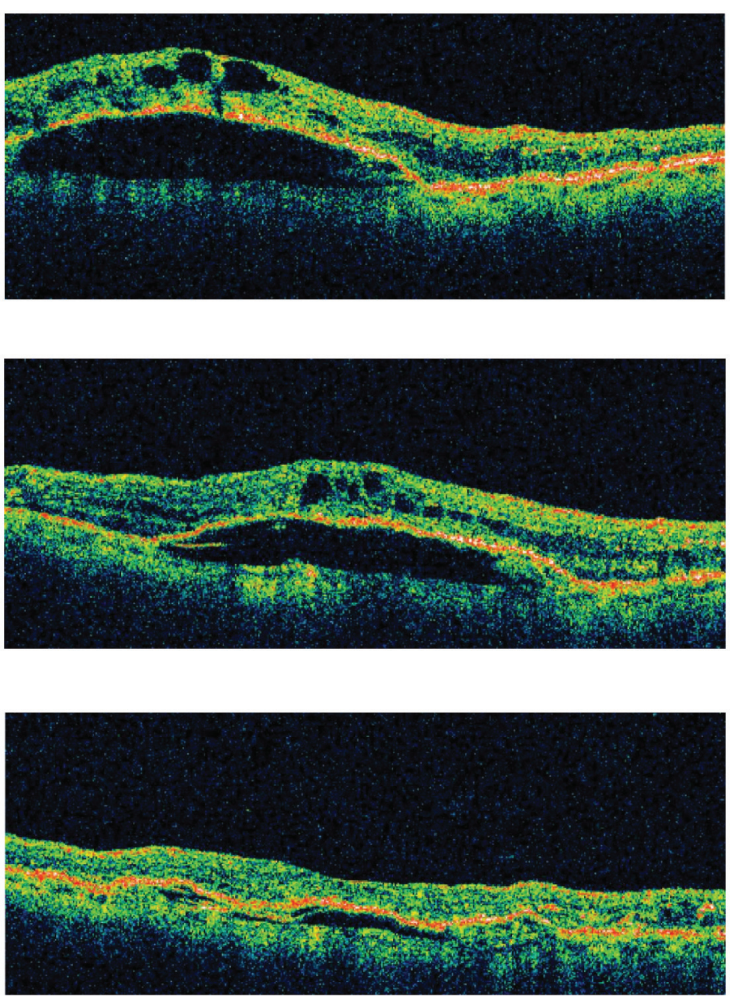

Figure 3 Representative optical coherence tomography (OCT) images of an eye with retinal angiomatous proliferation that had improvement in macular and subretinal pigment epithelial fluid between 4 and 8 weeks following a single intravitreal injection of bevacizumab. A) Baseline OCT images through central macula. B) OCT images at 4 weeks. C) OCT images at 8 weeks. 
Horizontal

A.

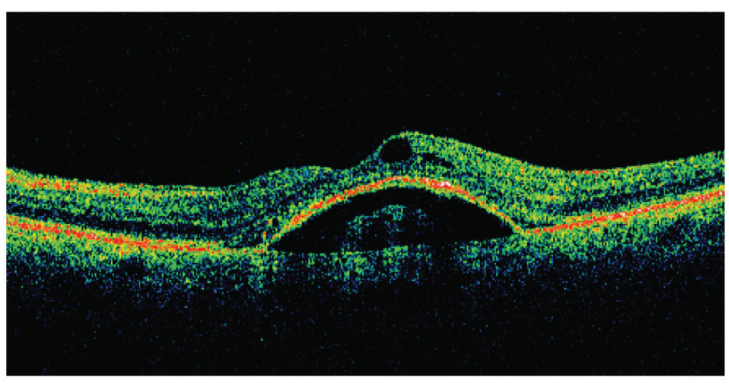

B.

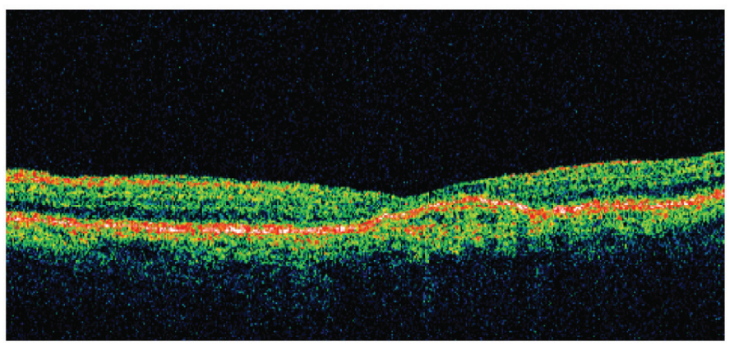

C.

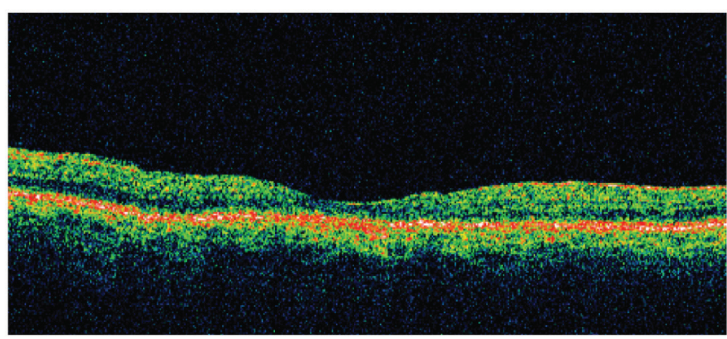

Vertical
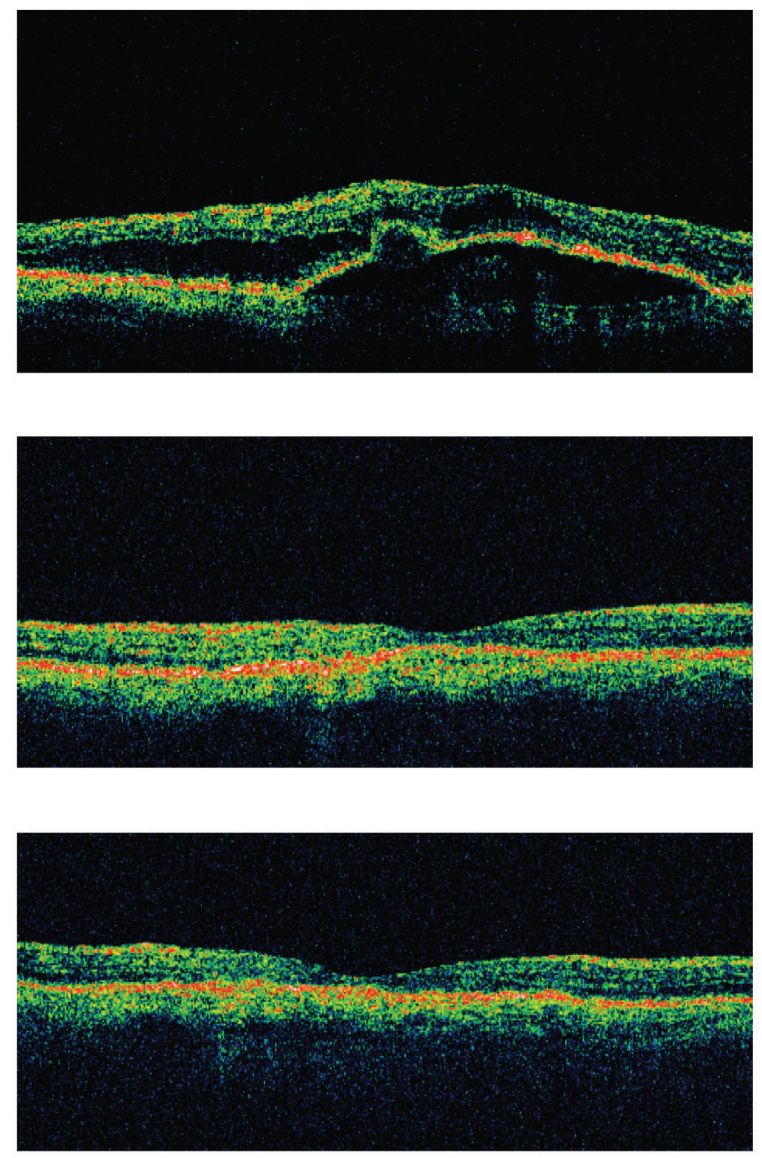

Figure 4 Representative optical coherence tomography (OCT) images of an eye with retinal angiomatous proliferation that had resolution of macular and subretinal pigment epithelial fluid at 4 weeks and maintained a dry macula at 8 weeks after a single intravitreal bevacizumab injection. A) Baseline OCT images through central macula. B) OCT images at 4 weeks. C) OCT images at 8 weeks.

Similarly, a macula that is dry at 4 weeks is very likely to remain dry at 8 weeks without additional treatment.

Accumulating clinical experience with as-needed or variable dosing regimens suggests that significant interindividual variation with respect to retreatment interval is a common feature of anti-VEGF therapy for neovascular AMD (Avery et al 2006; Rosenfeld et al 2006c; Fung et al 2007). However, extended follow-up of a cohort of patients treated with bevacizumab reinjection as needed for recurrent macular exudation found an average reinjection interval of 2 months during the first 6 months of treatment (Avery RL, Presented at The Combined Meeting of the Club Jules Gonin and The Retina Society, October, 2006; Cape Town, South Africa). Similarly, Aisenbrey and colleagues (2007) reported a median reinjection interval of 8 weeks in patients with exudative AMD who received bevacizumab reinjection as needed for worsening vision or exudation. In agreement with our data, these results suggest that most patients receiving intravitreal bevacizumab for exudative AMD can be followed initially at
6 to 8 week intervals, with subsequent titration of reinjection frequency depending on the individual clinical response.

Obviously, a dosing schedule that requires fewer injections provides significant advantages in terms of ocular safety, cost, and patient convenience and discomfort. Less frequent intravitreal dosing of a given drug also reduces systemic exposure to the drug and reduces the risk of systemic adverse events. Although encouraging, the results of this study must be interpreted in the context of its retrospective design, small number of patients, lack of standardized visual acuity testing, and short follow-up. Further research is necessary to provide further clarification of the ideal dosing strategy for bevacizumab and other anti-VEGF agents.

A planned prospective, randomized, multi-center clinical trial sponsored by the National Eye Institute (Comparison of Age-Related Macular Degeneration Treatments Trials: Lucentis-Avastin Trial, www.clinicaltrials.gov) will provide critical data comparing the efficacy and safety of ranibizumab with bevacizumab for neovascular AMD. Importantly, this 
trial will also compare fixed versus variable dosing schedules for each drug. Ultimately, this data will be needed to determine with certainty whether the benefits of less frequent dosing with bevacizumab can be realized without sacrificing the efficacy or safety proven with monthly ranibizumab.

\section{Disclosure}

The authors report no conflicts of interest in this work.

\section{References}

Aiello LP, Pierce EA, Foley ED, et al. 1995. Suppression of retinal neovascularization in vivo by inhibition of vascular endothelial growth factor (VEGF) using soluble VEGF-receptor chimeric proteins. Proc Natl Acad Sci USA, 92:10457-61.

Aisenbrey S, Ziemssen F, Volker M, et al. 2007. Intravitreal bevacizumab (Avastin) for occult choroidal neovascularization in age-related macular degeneration. Graefe's Arch Clin Exp Ophthalmol, 245:941-8.

Avery RL, Pieramici DJ, Rabena MD, et al. 2006. Intravitreal bevacizumab (Avastin) for neovascular age-related macular degeneration. Ophthalmology, 113:363-72.

Bashshur ZF, Bazarbachi A, Schakal A, et al. 2006. Intravitreal bevacizumab for the management of choroidal neovascularization in age-related macular degeneration. Am J Ophthalmol, 142:1-9.

Brown DM, Kaiser PK, Michels M, et al. 2006. Ranibizumab versus verteporfin for neovascular age-related macular degeneration. $N$ Engl $J$ Med, 355:1432-44.

Costa RA, Jorge R, Calucci D, et al. 2006. Intravitreal bevacizumab for choroidal neovascularization caused by AMD (IBeNA Study): Results of a phase 1 dose-escalation study. Invest Ophthalmol Vis Sci, 47:4569-78.

Fung AE, Lalwani GA, Rosenfeld PJ, et al. 2007. An optical coherence tomography-guided, variable dosing regimen with intravitreal ranibizumab (Lucentis) for neovascular age-related macular degeneration. Am J Ophthalmol, 143:566-83.

Gragoudas ES, Adamis AP, Cunningham Jr ET, et al. 2004. Pegaptanib for neovascular age-related macular degeneration. $N$ Engl $J$ Med, 351:2805-16.

Heier JS, Antoszyk AN, Pavan PR, et al. 2006. Ranibizumab for treatment of neovascular age-related macular degeneration: a phase I/II multicenter, controlled, multidose study. Ophthalmology, 113:633-42.
Kvanta A, Algvere PV, Berglin L, et al. 1996. Subfoveal fibrovascular membranes in age-related macular degeneration express vascular endothelial growth factor. Invest Ophthalmol Vis Sci, 37:1929-34.

Lopez PF, Sippy BD, Lambert HM, et al. 1996. Transdifferentiated retinal pigment epithelial cells are immunoreactive for vascular endothelial growth factor in surgically excised age-related macular degeneration-related choroidal neovascular membranes. Invest Ophthalmol Vis Sci, 37:8558-68.

Matsuoka M, Ogata N, Otsuji T, et al. 2004. Expression of pigment epithelium derived factor and vascular endothelial growth factor in choroidal neovascular membranes and polypoidal choroidal vasculopathy. Br J Ophthalmol, 88:809-15.

Michels S, Rosenfeld PJ, Puliafito CA, et al. 2005. Systemic bevacizumab (Avastin) therapy for neovascular age-related macular degeneration: Twelve-week results of an uncontrolled open-label clinical study. Ophthalmology, 112:1035-47.

Mordenti J, Cuthbertson RA, Ferrara N, et al. 1999. Comparisons of the intraocular tissue distribution, pharmacokinetics, and safety of 125Ilabeled full-length and Fab antibodies in rhesus monkeys following intravitreal administration. Toxicol Pathol, 27:536-44.

Rakic JM, Lambert V, Devy L, et al. 2003. Placental growth factor, a member of the VEGF family, contributes to the development of choroidal neovascularization, Invest Ophthalmol Vis Sci, 44:3186-93.

Rich RM, Rosenfeld PJ, Puliafito CA, et al. 2006. Short-term safety and efficacy of intravitreal bevacizumab (Avastin) for neovascular agerelated macular degeneration. Retina, 26:495-511.

Rosenfeld PJ, Moshfeghi AA, Puliafito CA. 2005. Optical coherence tomography findings after an intravitreal injection of bevacizumab (Avastin) for neovascular age-related macular degeneration. Ophthalmic Surg Lasers Imaging, 36:331-5.

Rosenfeld PJ, Brown DM, Heier JS et al. 2006a. Ranibizumab for neovascular age-related macular degeneration. $N$ Engl J Med, 355:1419-31.

Rosenfeld PJ, Heier JS, Hantsbarger G, et al. 2006b. Tolerability and efficacy of multiple escalating doses of ranibizumab (Lucentis) for neovascular age-related macular degeneration. Ophthalmology, 113:623-32.

Rosenfeld PJ, Rich RM, Lalwani GA. 2006c. Ranibizumab: Phase III clinical trial results. Ophthalmol Clin North Am, 19:361-72.

Sadde SR, Wu Z, Walsh AC, et al. 2006. Errors in retinal thickness measurements obtained by optical coherence tomography. Ophthalmology, 113:285-93

Spaide RF, Laud K, Fine HF, et al. 2006. Intravitreal bevacizumab treatment of choroidal neovascularization secondary to age-related macular degeneration. Retina, 26:383-90. 
